\title{
Cerebral revascularization for the management of complex middle cerebral artery aneurysm: A case series
}

\author{
XIAOCHENG LU* ${ }^{*}$ YABO HUANG ${ }^{*}$, PENG ZHOU, WEIWEI ZHU, ZHONG WANG and GANG CHEN \\ Department of Neurosurgery and Brain and Nerve Research Laboratory, The First Affiliated \\ Hospital of Soochow University, Suzhou, Jiangsu 215006, P.R. China
}

Received January 16, 2021; Accepted May 12, 2021

DOI: $10.3892 /$ etm.2021.10315

\begin{abstract}
Complex middle cerebral artery (MCA) aneurysms, including aneurysms that are sizeable (large or giant), fusiform, wide-necked or calcified, remain a significant challenge during microsurgical clipping or endovascular coiling as treatment strategies. In the present study, a retrospective analysis of cases of this type of aneurysm treated between August 2012 and December 2019 was performed. From the hospital's database, a total of 13 patients ( 7 males and 6 females) with a mean age of 39.0 years (range, 13-65 years) were identified. The mean size of the aneurysms was $17.5 \mathrm{~mm}$ (range, 3.9-35.0 $\mathrm{mm}$ ). A total of four patients $(30.8 \%)$ had ruptured aneurysms and nine $(69.2 \%)$ had unruptured aneurysms. All aneurysms were treated by proximal occlusion of the parent artery, trapping or excision combined with cerebral revascularization. The bypasses performed included 10 extracranial-intracranial bypasses and 3 intracranial-intracranial bypasses (1 end-to-end re-anastomosis, 1 interpositional graft and 1 end-to-side reimplantation). Postoperative angiography confirmed that the bypass patency was $92.3 \%$ and the clinical outcomes were indicated to be favorable, with a modified Rankin Scale score $\leq 2$ in 12 out of 13 patients $(92.3 \%)$ at the last follow-up. Taken together, the results of the present analysis suggested that treatment strategies for complex MCA aneurysms should depend on the status and characteristics of the aneurysm, including aneurysm size, location and morphology. For aneurysms that lack perforating arteries in the aneurysm dome, clip trapping or aneurysm excision with or without bypass are preferred as treatment strategies. When there are perforating
\end{abstract}

Correspondence to: Professor Yabo Huang, Department of Neurosurgery and Brain and Nerve Research Laboratory, The First Affiliated Hospital of Soochow University, 188 Shizi Street, Suzhou, Jiangsu 215006, P.R. China

E-mail:sdfyyhyb@126.com

*Contributed equally

Key words: complex middle cerebral artery aneurysm, high-flow bypass, low-flow bypass, surgical treatment arteries (particularly the lenticulostriate artery) arising from the aneurysm dome, however, the aneurysms should be treated with bypass followed by proximal occlusion of the parent artery or clip reconstruction.

\section{Introduction}

Recently, endovascular therapy has been proved as a safe alternative treatment strategy to surgical clipping for several intracranial aneurysms. However, in the field of middle cerebral artery (MCA) aneurysms, microsurgery remains superior to endovascular therapy, as these aneurysms are easily accessible and may be safely manipulated following splitting of the sylvian fissure (1-3). Furthermore, MCA aneurysms frequently have broad necks and branches often originate from the wall of the aneurysm, which may contribute to a higher risk of recurrence or retreatment with coiling and unintended occlusions with stent-assisted coiling $(4,5)$.

Complex MCA aneurysms, accounting for $4-17.4 \%$ of all MCA aneurysms, including aneurysms that are sizeable (large or giant), fusiform, wide-necked or feature intraluminal thrombi or incorporation of branches, remain a significant challenge to microsurgical clipping or endovascular coiling (6). Previous studies indicated that patients with complex MCA aneurysms had a mortality rate of $65-85 \%$ within 2 years due to rupture or re-rupture. Furthermore, survivors are frequently left with severe neurological deficits $(7,8)$. Complex MCA aneurysms are technically difficult to manage and a subset of them require management with bypass techniques as part of a treatment strategy with deliberate occlusion of a parent artery (9-13). Bypass techniques for MCA aneurysms include extracranial-to-intracranial (EC-IC) bypass, which comprises low-flow superficial temporal artery (STA)-M3 (or M4) bypass and high-flow external carotid artery-radial artery graft-M2 (ECA-RAG-M2) bypass, and intracranial-to-intracranial (IC-IC) bypass, including end-to-end reanastomosis, interpositional bypass, side-to-side anastomosis and reimplantation techniques. A study comprising 1,426 MCA aneurysms demonstrated that only $2.1 \%$ of MCA aneurysms required bypasses (14). To date, however, only a small number of studies have focused on cerebral revascularization for the treatment of complex MCA aneurysms (14-17). The present study reports on microsurgical treatment strategies for complex MCA aneurysms. 


\section{Materials and methods}

Study design and patient selection. The protocol of the present study was approved by the Institutional Review Board and Ethics committee of The First Affiliated Hospital of Soochow University (Suzhou, China). The medical records of all patients with complex MCA aneurysm treated using revascularization between August 2012 and December 2019 at The First Affiliated Hospital of Soochow University (Suzhou, China) were retrospectively reviewed. Complex MCA aneurysms were defined as those having been previously reported in the literature, including MCA aneurysms with a non-saccular morphology (dissecting, serpentine or fusiform) or with a large (10-24 $\mathrm{mm}$ in diameter) or a giant size ( $\geq 25 \mathrm{~mm}$ in diameter) or aberrant branch arteries arising from the side wall of the aneurysms (14). These aneurysms were considered complex when traditional treatment methods, such as clipping, stenting or coiling, were considered to be associated with high treatment risks. The patients' demographic and clinical data were reviewed, including patient age and sex, aneurysm characteristics (size, location and morphology), operative records, preoperative and postoperative images, complications, clinical outcomes and follow-up.

Surgical strategy. According to their locations, the aneurysms were divided into three subtypes: i) Prebifurcation; ii) bifurcation; and iii) postbifurcation. It was not possible to treat any of the MCA aneurysms included in the present study with direct clipping or clip reconstruction alone. The bypass types used in the present study were determined according to the preoperative aneurysm characteristics. The IC-IC bypass included the following subtypes: i) Reanastomosis (where the aneurysm was treated by excision and the arterial ends were sutured end-to-end); ii) interpositional bypass (where the aneurysm was excised and transected arterial ends were sutured with placement of an interposed graft); iii) reimplantation (with an end-to-side anastomosis between the transected end of the efferent artery of the aneurysm and an adjacent parallel donor artery) (6). The EC-IC bypass included STA-M3 (or M4) bypass (low-flow bypass) and the ECA-RAG-M2 bypass (high-flow bypass) (18).

The treatment strategy for each patient was determined by neurovascular surgeons and endovascular specialists, according to the aneurysm morphology, location, size and vascular anatomy. For aneurysms that lacked perforating arteries in the aneurysm dome, clip trapping the parent vessel with bypass was the preferred strategy. If there were perforating arteries [particularly the lenticulostriate artery (LSA)] arising from the aneurysm dome, these lesions were treated by proximal parent vessel occlusion with revascularization or clip reconstruction. The patency of the parent vessel and bypass were assessed by intra-operative Doppler flow probes and indocyanine green (ICG) angiography.

Imaging analysis and follow-up. Angiographic follow-up was performed using computed tomography (CT) angiography or digital subtraction angiography (DSA) to assess the bypass patency and aneurysm status 6 months postoperatively. The pre- and postoperative neurological outcomes (at 3, 6 and 12 months postoperatively) were assessed by the same author using the modified Rankin Scale (mRS) score (19).

\section{Results}

Patient characteristics. During a 7-year period between August 2012 and December 2019, 13 patients with 15 complex MCA aneurysms were surgically treated at The First Affiliated Hospital of Soochow University (Suzhou, China) (Table I). A total of 7 males and 6 females were included in the present study with an average age of 39.0 years (range, 13-65 years). In total, four patients presented with subarachnoid hemorrhage (SAH), of which three patients were Hunt and Hess grade II, and one patient was Hunt and Hess grade IV (20). Other presenting symptoms included headache, dizziness and epilepsy. Furthermore, one patient presented with a giant recurrent thrombotic aneurysm after a primary microsurgical clipping 8 years previously. The mean pretreatment mRS score was 1.4 (range, $1-5$ ).

The mean size of the 15 aneurysms was $17.5 \mathrm{~mm}$, with a range of 3.9-35.0 $\mathrm{mm}$. The locations of these aneurysms were classified as pre-bifurcation in three patients (20.0\%), bifurcation in five patients (33.3\%) and postbifurcation in seven patients $(46.7 \%)$.

Treatment with cerebral revascularization. All of the aneurysms were exposed through pterional craniotomy and a transsylvian approach (14). In total, seven aneurysms were managed by proximal occlusion of the parent artery, seven were managed by trapping and excision and one was managed by clip reconstruction plus partial occlusion. In total, 10 patients underwent EC-IC bypass, including six STA-MCA bypasses and four ECA-RAG-MCA bypasses. The IC-IC bypass treatments included one reanastomosis, one interpositional bypass and one reimplantation.

Surgical results and follow-up. No mortality occurred as a result of surgery. The mean follow-up time was 28.3 months (range, 9-97 months). The mean mRS score was 0.8 (range, $0-3$ ) at 3 months postoperatively. The condition of all patients either improved or was equal to that of the preoperative state except for one patient, whose postoperative $\mathrm{mRS}$ score increased from 1 to 2 (Table I). In total, 92.3\% of the bypasses (12/13) were patent, which was confirmed by postoperative angiography at 6 months postoperatively. The angiographic images indicated that 9 of the 10 EC-IC bypasses were patent (90.0\%) and the IC-IC bypass was patent in all patients at follow-up. The bypass was occluded in one patient, which was indicated by the angiography at 6 months postoperatively. However, the aneurysm almost disappeared and there were no neurological deficits. Thus, no further treatment was needed for this patient. There was no recurrence or mortality during the follow-up period.

\section{Illustrative cases}

Case I. A 40-year-old female presented with headache (Fig. 1; case 12 in Table I). A CT scan revealed a space-occupying lesion in the right sylvian fissure. The CT scan and angiography (CTA) and DSA results revealed the presence of a giant fusiform aneurysm originating from the right M3 segment (diameter, $20.0 \mathrm{~mm}$ ) (Fig. 1A). The subsequent 3D reconstructive DSA indicated that the aneurysm had one afferent artery (proximal M3) and two efferent arteries (the distal M3 and 


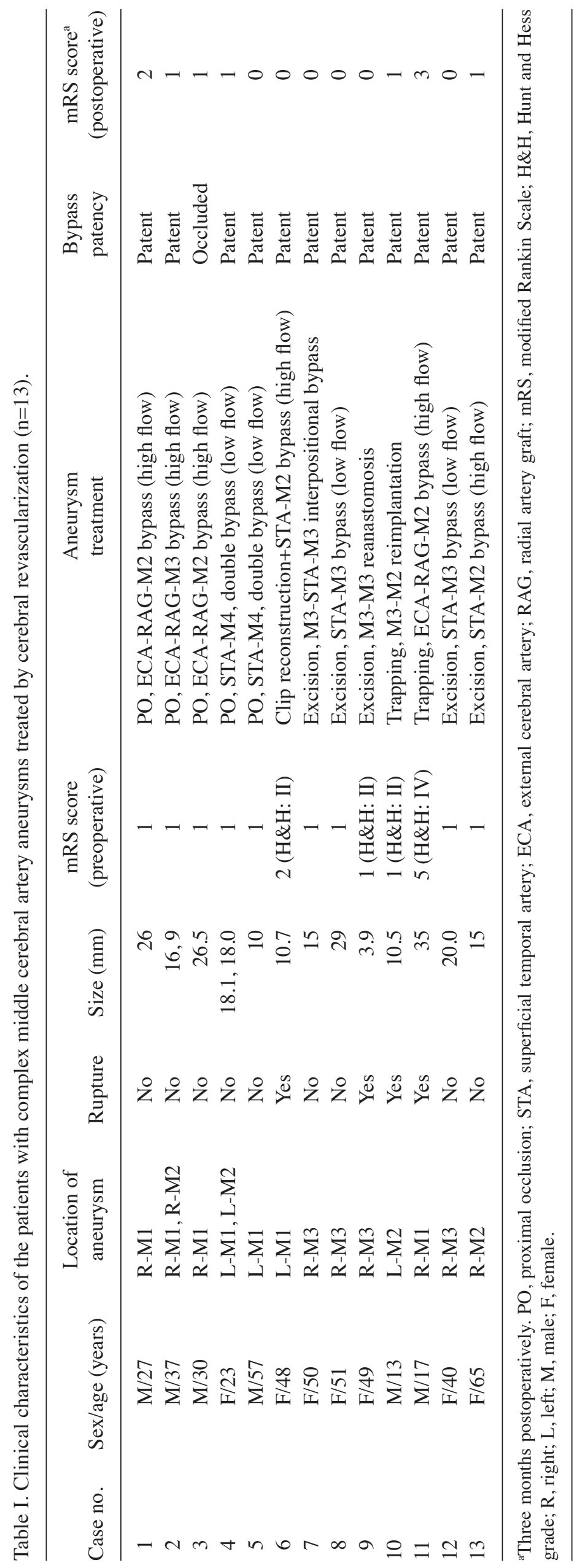



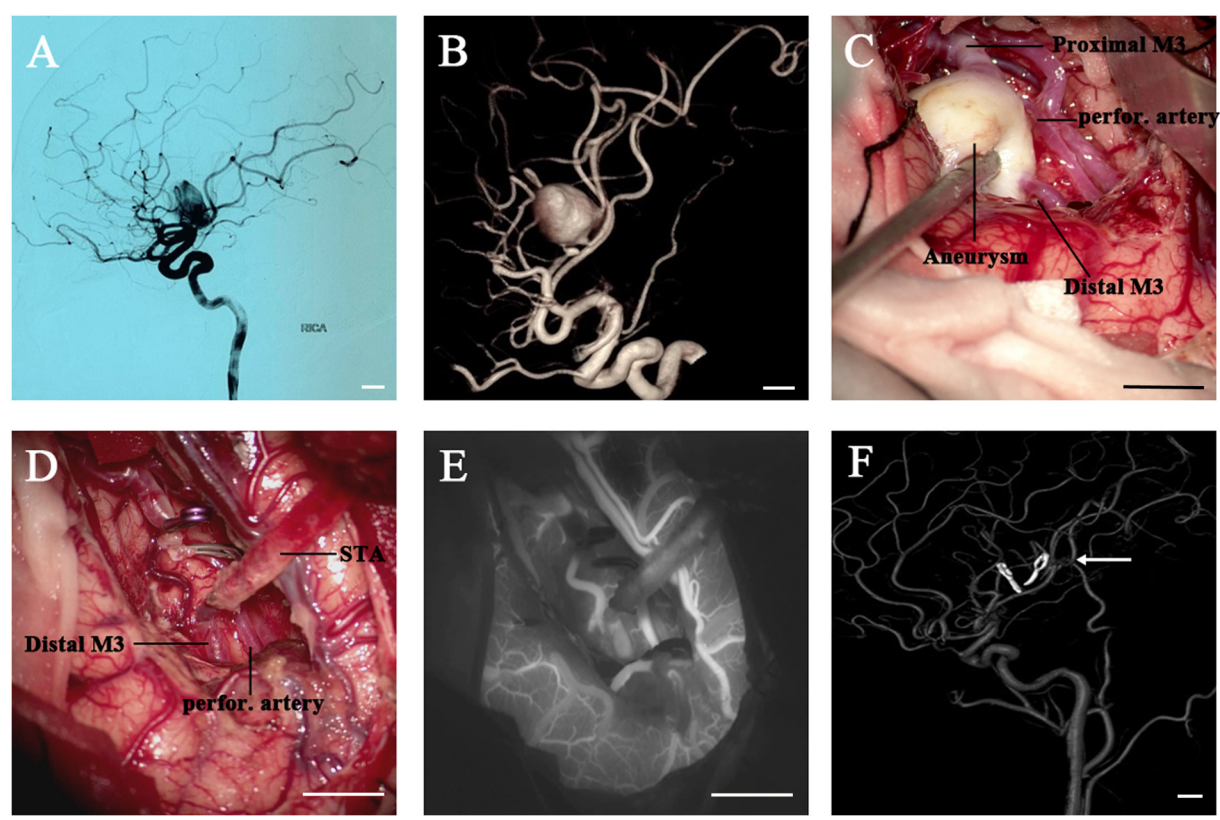

Figure 1. Images from a 40-year-old female who presented with a headache. (A) The DSA image (RICA injection, lateral view) and (B) 3D reconstruction displayed a large fusiform aneurysm originating from the right M3 segment. (C and D) Intraoperative images. (C) The aneurysm was confirmed during the operation with one afferent artery (proximal M3) and two efferent arteries (the distal M3 and a small perforating artery arising from the aneurysm dome). (D) An STA-M3 end-to-end anastomosis was performed, followed by excision of the aneurysm. (E) The intraoperative indocyanine green videoangiography indicated simultaneous filling of the distal M3 and the perforating artery. (F) The 3D reconstruction of postoperative DSA (right common carotid artery injection) confirmed complete obliteration of the aneurysm and patency of the bypass (white arrow). Scale bars, $10 \mathrm{~mm}$. DSA, digital subtraction angiography; RICA, right internal carotid artery; STA, superficial temporal artery; perfor., perforating.
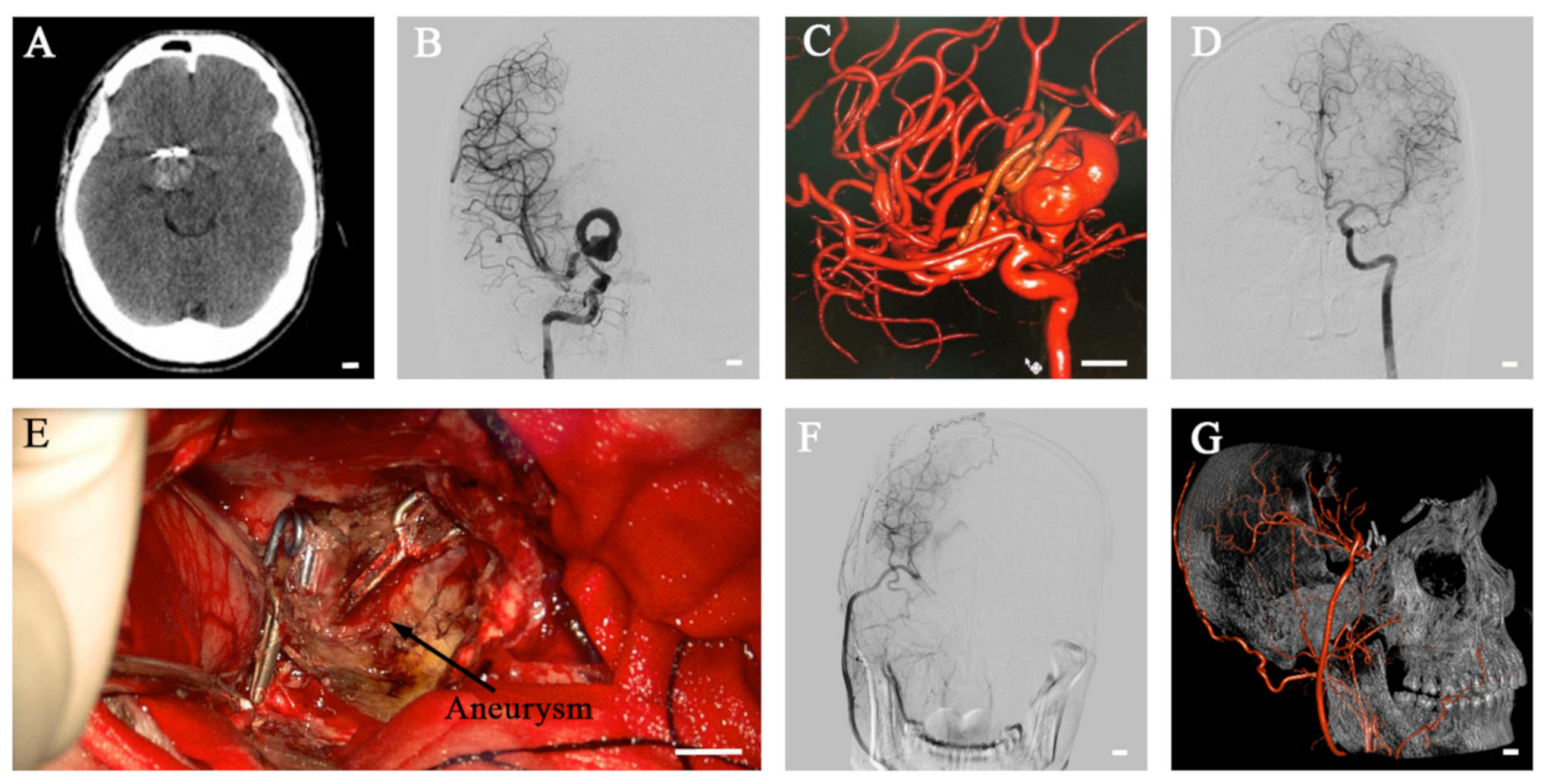

Figure 2. Images obtained from a 27-year-old male with a giant recurrent thrombotic aneurysm originating from the right M1 segment. (A) Axial CT scan indicating a recurrent giant thrombotic right middle cerebral artery aneurysm. (B) DSA image (right ICA injection, anteroposterior view) and (C) 3D reconstruction, which confirmed the aneurysm prior to surgery. (D) DSA image revealing that the right A2 was supplied by the left A1 via the anterior communicating artery. (E) The aneurysm was confirmed during surgery and treated with proximal occlusion with a right external carotid artery-radial artery-M2 bypass (F) The DSA image (right common carotid artery injection, anteroposterior view) confirmed the exclusion of the aneurysm and patency of the bypass. (G) The 3D reconstruction of the postoperative DSA image (left ICA injection, lateral view) displayed the exclusion of the aneurysm and patency of the bypass. Scale bars, $10 \mathrm{~mm}$. DSA, digital subtraction angiography; ICA, internal carotid artery.

a small perforating artery arising from the aneurysm dome) (Fig. 1B), which was confirmed during the operation. (Fig. 1C) The aneurysm was exposed using the right pterional approach. Following STA-M3 end-to-end anastomosis, the proximal and distal M3 segment were temporarily occluded. Since aneu- rysm excision was impossible without sacrificing the small perforating artery arising from the aneurysm, intra-operative ICG videoangiography was used to determine whether there was any collateral circulation to provide an adequate blood supply for the perforating artery (Fig. 1D). ICG videoan- 

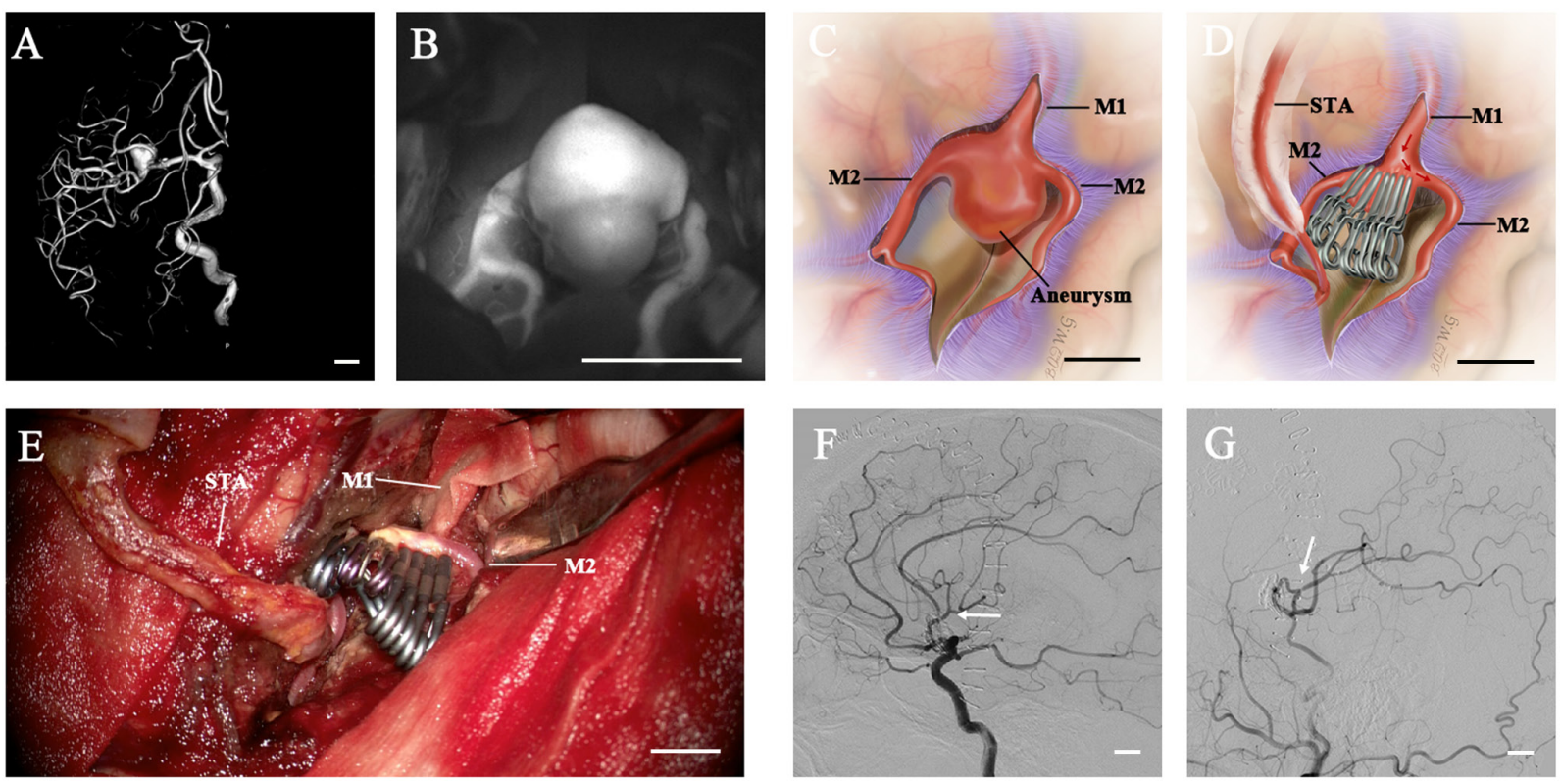

Figure 3. Images from a 48-year-old female with a fusiform aneurysm originating from the left M1 bifurcation. (A) The 3D reconstruction of the DSA image (left ICA injection, oblique view) indicated a fusiform aneurysm located M1 bifurcation with two M2 trunks involved. (B) The indocyanine green videoangiography and (C) a supporting diagram revealing that the M1 afferent artery, the inferior M2 trunk arising from the aneurysm and the superior M2 trunk originating from the base of the aneurysm were not involved in the neck of the aneurysm. (D) A diagram and (E) intraoperative image displaying the surgical procedure. Tandem clipping was applied to reconstruct the middle cerebral artery bifurcation and preserve the superior M2 trunk. The aneurysm between the M1 segment and inferior trunk was completely obliterated and an STA-M2 bypass was performed to supply anterograde flow to the inferior M2 trunk. (F) The postoperative DSA image (left ICA injection, lateral view) confirmed that the aneurysm was completely obliterated and the reconstructive M1-superior M2 trunk was patent (as indicated by the white arrow). (G) The postoperative DSA (left external carotid artery injection, lateral view) indicated the patency of the STA-M2 (inferior) bypass (white arrow). Scale bars, $10 \mathrm{~mm}$. DSA, digital subtraction angiography; ICA, internal carotid artery; STA, superficial temporal artery.

giography revealed simultaneous filling of the distal M3 and the perforating arteries for this patient (Fig. 1E), indicating sufficient blood supply. Therefore, the aneurysm was resected and no further processing was necessary. After the surgery, the patient was free of any neurological deficits and the postoperative DSA demonstrated a patent bypass and complete exclusion of the aneurysm (6 months after the operation) (Fig. 1F).

Case II. A 27-year-old male suffered from numbness of the left limbs for 1 month and had received microsurgical clipping of a right MCA aneurysm 8 years previously (Fig. 2; case 1 in Table I). A CT scan revealed a giant lesion in the right front-temporal lobe (Fig. 2A). A subsequent DSA assessment demonstrated a giant recurrent thrombotic aneurysm originating from the right M1 segment and direct clipping or endovascular treatment was therefore impossible (Fig. 2B-D). The aneurysm was treated with proximal occlusion and a right ECA-RA-M2 bypass was performed to supply retrograde flow to the non-bypassed M2 artery and LSA (Fig. 2E). Intraoperative ICG videoangiography and Doppler ultrasonography confirmed the patency of the bypass. The postoperative DSA demonstrated complete obliteration of the aneurysm (Fig. 2F and G). However, the postoperative mRS score increased from 1 (preoperative) to 2 (postoperative) with decreased myodynamia of the right limb, which may have occurred since the ECA-RA-M2 bypass was not able to sufficiently support the MCA territory.

Case III. A 48-year-old female presented with a sudden headache and was referred to our department (Fig. 3; case 6 in Table I). A CT scan revealed SAH in the right sylvian fissure. The CTA and DSA analyses revealed a fusiform aneurysm originating from the left M1 bifurcation (Fig. 3A). After the aneurysm had been exposed, the intraoperative observation was that the inferior M2 trunk had arisen from the aneurysm, making direct clipping impossible. The superior M2 trunk was not involved in the neck of the aneurysm (Fig. 3B-D). Following temporary occlusion, the aneurysm was opened, the aneurysmal neck was completely transected to simplify the clipping and the intraluminal thrombus was removed. Tandem clipping with stacked straight fenestrated clips was applied to reconstruct the MCA bifurcation and preserve the superior trunk. A straight clip was used to completely obliterate the aneurysm between the M1 segment and inferior trunk and an STA-M2 bypass was performed to supply anterograde flow to the inferior trunk (Fig. 3E). The patient recovered well without any neurological deficits. Postoperative DSA demonstrated complete occlusion of the aneurysm, a reconstructive superior M2 trunk and patency of the STA-M2 (inferior) bypass (Fig. 3F and G).

\section{Discussion}

MCA aneurysms have long been considered favorable for clipping since they are easily accessible followed by splitting of the sylvian fissure. Complex MCA aneurysms usually possess unfavorable features, including intraluminal thrombi, fusiform configurations, mural calcifications and the incorporation of branches (21-23). These features limit the feasibility of direct clipping or endovascular coiling alone. In the present study, various cerebral revascularization techniques used to treat complex MCA aneurysms during a 7-year period were 
described. The analysis of 13 patients with complex MCA aneurysms revealed that the patency rate was $92.3 \%$ and 12 of 13 aneurysms were removed from the circulation. All patients had a favorable outcome $(\mathrm{mRS} \leq 2)$ at the last follow-up.

Several surgical techniques have been reported for the treatment of complex MCA aneurysms, including clip reconstruction, aneurysmal excision with a reanastomosis, proximal occlusion or surgical trapping with or without cerebral revascularization $(11,14,17,18)$. Clip reconstruction is a commonly used direct occlusion strategy for the treatment of complex MCA aneurysm with broad necks or giant size and this is mediated through molding the neck, shrinking the aneurysm with low-flow electrocoagulation or reconstructing the efferent arteries $(24,25)$. In the present study, a giant fusiform aneurysm located in the M1 bifurcation was managed with clip reconstruction and EC-IC bypass. Tandem clipping with stacked straight fenestrated clips was used to reconstruct the M1/superior M2 antegrade tube and STA-M2 bypass was applied to supply anterograde flow to the inferior M2 trunk (case III). However, clip reconstruction was impossible to perform in the majority of cases of the present study, since most complex MCA aneurysms had a dolichoectatic morphology or atherosclerotic necks.

Indirect occlusion with or without cerebral revascularization remains the most important treatment strategy for complex MCA aneurysms, which includes aneurysmal trapping, aneurysmal excision, proximal occlusion or distal occlusion of the parent artery. Complete trapping, exclusion of the aneurysm and the total arterial territory may result in ischemic complications when perforating arteries arise from the aneurysm. Therefore, complete trapping or excision of the aneurysm plus IC-IC or EC-IC bypass is an appropriate surgical strategy for the treatment of complex MCA aneurysms located in the distal segment of the MCA and that lack aberrant branch arteries or perforating arteries arising from the aneurysm. In the present study, three patients were treated with aneurysm trapping or excision followed by IC-IC bypass, of which two aneurysms were treated by excision combined with M3-M3 reanastomosis or M3-STA-M3 reanastomosis, respectively. The other one was treated with trapping and M3-M2 reimplantation.

Previous studies suggested that IC-IC bypass has aneurysm obliteration rates, bypass patency rates and complication rates similar to those of EC-IC bypass (26). However, anatomical constraints, such as an aneurysm in the insular recess, may limit the IC-IC bypass option (27). In addition, IC-IC bypass is generally suitable for distally located aneurysms, as the large hemispheric territory supplied by the MCA requires high-flow bypass in prebifurcation or bifurcation aneurysms (27). EC-IC bypass is also a well-established strategy for the treatment of complex aneurysms. It is appropriate for pre-bifurcation and bifurcation MCA aneurysm, as, unlike a distal efferent artery in postbifurcation aneurysm, a proximal efferent artery calls for high flow (27). In the present study, 10 patients were treated with proximal occlusion of the parent artery, trapping or excision of aneurysms combined with EC-IC bypass, including 4 ECA-RAG-MCA bypasses and 6 STA-MCA bypasses.

Complete trapping or excision of the aneurysm may be the best strategy for treating aneurysms free of perforating vessels (6). However, in complex MCA aneurysms, the aneurysm cannot be trapped or excised when the LSA or aberrant branch arteries originate from the aneurysm and in these cases, proximal occlusion is performed instead. In the present study, six complex MCA aneurysms were managed with proximal occlusion of the parent artery plus bypass. Proximal occlusion, a subtype of partial trapping, is able to reduce the flow through the aneurysm to facilitate intra-aneurysmal thrombosis and the bypass provides anterograde flow to the recipient trunk and retrograde blood flow for the perforators (18). Despite the perforating arteries, the rupture status of the aneurysm also affects the type of parent arterial occlusion. Ruptured MCA aneurysms should be excluded completely with direct neck clipping or trapping together with EC-IC/IC-IC bypass when clipping is impossible, since retrograde filling of a ruptured aneurysm may lead to rerupture of the aneurysm (18).

Recently, flow diversion devices have been reported as an alternative endovascular treatment for complex MCA aneurysms. Cimflova et al (28) reported that the complete/near-complete occlusion rate was $70 \%$ in consecutive subjects with $23 \mathrm{MCA}$ aneurysms. Potentially severe periprocedural or postprocedural events occurred in $17.4 \%$ of the patients and severe complications (intraparenchymal hemorrhage and rerupture of the aneurysm) occurred in half of those patients $(8.7 \%)$, which was consistent with previous studies $(29,30)$. Furthermore, the requirement for antiplatelet agents limits their applicability in patients with a history of SAH. However, managing complex MCA aneurysms with a combined approach of coil embolization following protective EC-IC bypass has been reported in a recent study (31), which may provide an alternative treatment strategy for complex MCA aneurysms, particularly when surgical trapping or proximal occlusion is impossible due to anatomic limitations.

Of note, the present study had certain limitations. First, the results are limited since the experience reported was from a single surgeon at a single center, which may not be representative of generalized neurosurgical results. Moreover, the follow-up period of the present study was relatively short, and the surgical strategies were based to the limited number of patients.

In conclusion, surgical treatment strategies for complex MCA aneurysm should depend on the status and characteristics of the aneurysm, including aneurysm size, location and morphology, and particularly the involvement of perforating arteries. Favorable outcomes may be achieved with appropriate surgical strategies.

\section{Acknowledgements}

Not applicable.

\section{Funding}

This work is supported by the National Natural Science Foundation of China (grant no. 81601064). The funders had no role in the preparation of this study or interpretation of the results.

\section{Availability of data and materials}

The datasets used and/or analyzed during the present study are available from the corresponding author on reasonable request. 


\section{Authors' contributions}

YH, PZ and XL contributed to surgical treatment and drafted the manuscript. WZ performed endovascular interventions. PZ, XL, GC and ZW participated in case management, data extraction and data analysis. YH and XL confirmed the authenticity of the raw data. All authors read and approved the final manuscript.

\section{Ethics approval and consent to participate}

All procedures performed in studies involving human participants were in accordance with the ethical standards of the First Affiliated Hospital of Soochow University (Suzhou, China) and with the 1964 Helsinki declaration and its later amendments or comparable ethical standards. Written informed consent was obtained from the participants.

\section{Patient consent for publication}

The patients provided consent for publication. There were two patients under the legal age of consent and consent was obtained from their parents.

\section{Competing interests}

The authors declare that they have no competing interests.

\section{References}

1. Smith TR, Cote DJ, Dasenbrock HH, Hamade YJ, Zammar SG, El Tecle NE, Batjer HH and Bendok BR: Comparison of the efficacy and safety of endovascular coiling versus microsurgical clipping for unruptured middle cerebral artery aneurysms: a systematic review and meta-analysis. World Neurosurg 84: 942-953, 2015.

2. Diaz OM, Rangel-Castilla L, Barber S, Mayo RC, Klucznik R and Zhang YJ: Middle cerebral artery aneurysms: A single-center series comparing endovascular and surgical treatment. World Neurosurg 81: 322-329, 2014.

3. Steklacova A, Bradac O, Charvat F, De Lacy P and Benes V: 'Clip first' policy in management of intracranial MCA aneurysms: Single-centre experience with a systematic review of literature. Acta Neurochir (Wien) 158: 533-546, discussion 546, 2016.

4. Lu P, Zhang Y, Niu H and Wang Y: Comparison of endovascular treatment for middle cerebral artery aneurysm with a low-profile visualized intraluminal support stent or pipeline embolization device. Exp Ther Med 18: 2072-2078, 2019.

5. Jeong SM, Kang SH, Lee NJ and Lim DJ: Stent-assisted coil embolization for the proximal middle cerebral artery fusiform aneurysm. J Korean Neurosurg Soc 47: 406-408, 2010.

6. Nussbaum ES, Kallmes KM, Lassig JP, Goddard JK, Madison MT and Nussbaum LA: Cerebral revascularization for the management of complex intracranial aneurysms: A single-center experience. J Neurosurg: Oct 1, 2018 (Epub ahead of print).

7. Choi IS and David C: Giant intracranial aneurysms: Development, clinical presentation and treatment. Eur J Radiol 46: 178-194, 2003

8. Drake CG and Peerless SJ: Giant fusiform intracranial aneurysms: Review of 120 patients treated surgically from 1965 to 1992. J Neurosurg 87: 141-162, 1997.

9. Fulkerson DH, Voorhies JM, Payner TD, Leipzig TJ, Horner TG, Redelman K and Cohen-Gadol AA: Middle cerebral artery aneurysms in children: Case series and review. J Neurosurg Pediatr 8: 79-89, 2011.

10. Quiñones-Hinojosa A and Lawton MT: In situ bypass in the management of complex intracranial aneurysms: Technique application in 13 patients. Neurosurgery 57 (Suppl 1): 140-145, discussion 140-145, 2005

11. Sekhar LN, Stimac D, Bakir A and Rak R: Reconstruction options for complex middle cerebral artery aneurysms. Neurosurgery 56 (Suppl 1): 66-74, discussion 66-74, 2005.
12. van Doormaal TP, van der Zwan A, Verweij BH, Han KS, Langer DJ and Tulleken CA: Treatment of giant middle cerebral artery aneurysms with a flow replacement bypass using the excimer laser-assisted nonocclusive anastomosis technique. Neurosurgery 63: 12-20, discussion 20-22, 2008.

13. Aboukais R, Verbraeken B, Leclerc X, Gautier C, Vermandel M, Bricout N, Lejeune JP and Menovsky T: Protective STA-MCA bypass to prevent brain ischemia during high-flow bypass surgery: Case series of 10 patients. Acta Neurochir (Wien) 161: 1207-1214, 2019.

14. Tayebi Meybodi A, Huang W, Benet A, Kola O and Lawton MT: Bypass surgery for complex middle cerebral artery aneurysms: An algorithmic approach to revascularization. J Neurosurg 127: 463-479, 2017.

15. Lan J, Fu ZY, Zhang JJ, Ma C, Cao CJ, Zhao WY, Jiang PC and Chen JC: Giant serpentine aneurysm of the middle cerebral artery. World Neurosurg 117: 109-114, 2018.

16. Kivipelto L, Niemelä M, Meling T, Lehecka M, Lehto H and Hernesniemi J: Bypass surgery for complex middle cerebral artery aneurysms: Impact of the exact location in the MCA tree. J Neurosurg 120: 398-408, 2014.

17. Wang L, Lu S, Cai L, Qian H, Tanikawa R and Shi X: Internal maxillary artery bypass for the treatment of complex middle cerebral artery aneurysms. Neurosurg Focus 46: E10, 2019.

18. Xu F, Xu B, Huang L, Xiong J, Gu Y and Lawton MT: Surgical Treatment of Large or Giant Fusiform Middle Cerebral Artery Aneurysms: A Case Series. World Neurosurg 115: e252-e262, 2018.

19. Banks JL and Marotta CA: Outcomes validity and reliability of the modified Rankin scale: implications for stroke clinical trials: a literature review and synthesis. Stroke 38: 1091-1096, 2007.

20. Konczalla J, Seifert V, Beck J, Güresir E, Vatter H, Raabe A and Marquardt G: Outcome after Hunt and Hess Grade V subarachnoid hemorrhage: A comparison of pre-coiling era (1980-1995) versus post-ISAT era (2005-2014). J Neurosurg 128: 100-110, 2018.

21. al-Yamany M and Ross IB: Giant fusiform aneurysm of the middle cerebral artery: Successful Hunterian ligation without distal bypass. Br J Neurosurg 12: 572-575, 1998.

22. Rodríguez-Hernández A, Sughrue ME, Akhavan S, Habdank-Kolaczkowski J and Lawton MT: Current management of middle cerebral artery aneurysms: Surgical results with a 'clip first' policy. Neurosurgery 72: 415-427, 2013.

23. Heros RC and Fritsch MJ: Surgical management of middle cerebral artery aneurysms. Neurosurgery 48: 780-785, discussion 785-786, 2001.

24. Kim LJ, Klopfenstein JD and Spetzler RF: Clip reconstruction and sling wrapping of a fusiform aneurysm: Technical note. Neurosurgery 61 (Suppl 3): 79-80, discussion 80, 2007.

25. Yang I and Lawton MT: Clipping of complex aneurysms with fenestration tubes: Application and assessment of three types of clip techniques. Neurosurgery 62 (Suppl 2): ONS371-ONS378, discussion 378-379, 2008.

26. Sanai N, Zador Z and Lawton MT: Bypass surgery for complex brain aneurysms: An assessment of intracranial-intracranial bypass. Neurosurgery 65: 670-683, discussion 683, 2009.

27. Zhu W, Liu P, Tian Y, Gu Y, Xu B, Chen L, Zhou L and Mao Y: Complex middle cerebral artery aneurysms: A new classification based on the angioarchitecture and surgical strategies. Acta Neurochir (Wien) 155: 1481-1491, 2013.

28. Cimflova P, Özlük E, Korkmazer B, Ahmadov R, Akpek E, Kizilkilic O, Islak C and Kocer N: Long-term safety and efficacy of distal aneurysm treatment with flow diversion in the M2 segment of the middle cerebral artery and beyond. J Neurointerv Surg: Oct 20, 2020 (Epub ahead of print).

29. Topcuoglu OM, Akgul E, Daglioglu E, Topcuoglu ED, Peker A, Akmangit I, Belen D and Arat A: Flow diversion in middle cerebral artery aneurysms: is it really an all-purpose treatment? World Neurosurg 87: 317-327, 2016.

30. Zanaty M, Chalouhi N, Tjoumakaris SI, Gonzalez LF, Rosenwasser R and Jabbour P: Flow diversion for complex middle cerebral artery aneurysms. Neuroradiology 56: 381-387, 2014.

31. Shi ZS, Ziegler J, Duckwiler GR, Jahan R, Frazee J, Ausman JI, Martin NA and Viñuela F: Management of giant middle cerebral artery aneurysms with incorporated branches: Partial endovascular coiling or combined extracranial-intracranial bypass - a team approach. Neurosurgery 65 (Suppl 6): 121-129, discussion 129-131, 2009.

This work is licensed under a Creative Commons

Attribution-NonCommercial-NoDerivatives 4.0 International (CC BY-NC-ND 4.0) License. 